\title{
Self-consistent Green's-function technique for surfaces and interfaces
}

\section{Skriver, Hans Lomholt; Rosengaard, N. M.}

\section{Published in:}

Physical Review B

Link to article, DOI:

10.1103/PhysRevB.43.9538

Publication date:

1991

Document Version

Publisher's PDF, also known as Version of record

Link back to DTU Orbit

Citation (APA):

Skriver, H. L., \& Rosengaard, N. M. (1991). Self-consistent Green's-function technique for surfaces and interfaces. Physical Review B, 43(12), 9538-9549. https://doi.org/10.1103/PhysRevB.43.9538

\section{General rights}

Copyright and moral rights for the publications made accessible in the public portal are retained by the authors and/or other copyright owners and it is a condition of accessing publications that users recognise and abide by the legal requirements associated with these rights.

- Users may download and print one copy of any publication from the public portal for the purpose of private study or research.

- You may not further distribute the material or use it for any profit-making activity or commercial gain

- You may freely distribute the URL identifying the publication in the public portal

If you believe that this document breaches copyright please contact us providing details, and we will remove access to the work immediately and investigate your claim 


\title{
Self-consistent Green's-function technique for surfaces and interfaces
}

\author{
H. L. Skriver and N. M. Rosengaard \\ Laboratory of Applied Physics, \\ Technical University of Denmark, DK-2800 Lyngby, Denmark
}

(Received 21 December 1990)

\begin{abstract}
We have implemented an efficient self-consistent Green's-function technique for calculating ground-state properties of surfaces and interfaces, based on the linear-muffin-tin-orbitals method within the tight-binding representation. In this approach the interlayer interaction is extremely short ranged, and only a few layers close to the interface need be treated self-consistently via a Dyson equation. For semi-infinite jellium, the technique gives work functions and surface energies that are in excellent agreement with earlier calculations. For the bcc(110) surface of the alkali metals, we find surface energies in close agreement with values derived from surface tensions of the liquid metals, and work functions that deviate less than $10 \%$ from the experimental values.
\end{abstract}

\section{INTRODUCTION}

In order to evaluate the physical properties of surfaces and interfaces, it is important to take proper account of the breakdown of translational symmetry that occurs perpendicular to the interface. In the past this has very often been done in an approximate fashion by means of standard one-electron methods applied to either a supercell or a slab geometry. While these methods will give accurate results when carried to convergence in the number of layers, they need extremely large computer resources, and hence no comprehensive study of, for instance, work functions of metals from first principles has yet been undertaken.

A different and more natural approach to the interface problem would be to use a Green's-function technique. In such an approach, one deals directly with the electronic states in a semi-infinite crystal having only a single interface. This is in contrast to the usual slab or supercell methods, where the symmetry forces one to treat two interfaces simultaneously. During the past two decades several Green's-function techniques for surfaces and interfaces have been proposed, ${ }^{1-5}$ and the formalisms have been thoroughly developed as far as formal aspects are concerned. However, applications so far have been limited mostly to empirical tight-binding and non-self-consistent muffin-tin models, and it is only recently, when the techniques were combined with localdensity theory, that self-consistent results have been forthcoming. ${ }^{6,7}$

In this paper we describe and present the first applications of a new and efficient, self-consistent Green'sfunction technique for structures with only two-dimensional periodicity, based on the generalized linear-muffintin-orbitals (LMTO) method. ${ }^{8-10}$ A very similar approach has been used successfully for point defects, ${ }^{11-13}$ while an outline of part of the present scheme together with some preliminary results were given by Lambrecht and Andersen. ${ }^{14}$

The generalized LMTO method is presented in depth in the papers by Andersen and co-workers. ${ }^{8-10}$ In this paper, we shall therefore only be concerned with those details of the theory that are important for our Green'sfunction technique. As to exposition and notation we shall follow closely the presentation given in Ref. 10, and we shall take full advantage of the theory of linear transformations between generalized MTO's in different representations. Here, a representation is specified by a particular choice of the matrix $\alpha$ used in the screening calculation for the structure constants. In particular, we shall make use of three representations, the conventional ( $\alpha=0$ ), the orthogonal $(\alpha=\gamma)$, and the most localized; tight binding $(\alpha=\beta)$.

One of the most important aspects of the present technique is the ability, within the atomic-sphere approximation (ASA) and in the tight-binding representation, to generate the Green's-function matrices for a real, twodimensional interface by a simple and efficient procedure. In the present work we stay entirely within the ASA as far as the potential is concerned, but for the charge density we include the dipole moments. If the accuracy of this approximation is inadequate, e.g., if nonspherical contributions to the atomic-sphere potential are important, one may transform to a Hamiltonian Green'sfunction representation and proceed as in a conventional Green's-function calculation. The present technique will then serve as an extremely convenient way of obtaining a Green's function for a real interface.

\section{LMTO GREEN'S-FUNCTION METHOD FOR INTERFACES}

Our adaptation of the LMTO method to the interface problem is naturally separated into several distinct steps. The starting point is formed by standard band calcula- 
tions and subsequent construction of the infinite-crystal Green's-function matrices for the two types of solids that form the interface under consideration.

From these we construct the Green's-function matrices for the left- and right-hand semi-infinite sides of the interface by a Löwdin downfolding technique. In the case of a surface, the semi-infinite Green's-function matrices for the vacuum region are obtained by an analytical expression.

In the next step, the semi-infinite Green's-function matrices for the two sides are combined in a process inverse to the Löwdin downfolding to form Green's-function matrices for an ideal interface. Here, ideal means that the atomic positions and the electronic densities are unrelaxed.

These ideal-interface Green's-function matrices are subsequently used as the zeroth-order Green's functions in a Dyson equation for those few atomic layers close to the interface which must be treated self-consistently. When the Dyson equation is iterated to self-consistency, we have all the quantities needed to estimate physical properties, such as work functions and surface energies, connected with the interface.

\section{A. The Green's functions}

In the following sections, we present the various Green's-function matrices that enter our interface technique and we describe in detail how they are obtained numerically.

\section{The initial calculations}

At the outset of an interface calculation, one would like to have appropriate starting potentials as well as total energies corresponding to perfect, infinite crystals of the atomic species that are brought together to form the interface. To obtain this input, we perform self-consistent energy-band calculations by means of the conventional LMTO method within the atomicsphere approximation. ${ }^{15}$ In the actual calculations we apply the most recent versions of the programs described by Skriver, ${ }^{16}$ and use their output to start the Green'sfunction calculations.

\section{Hamiltonian Green's function for the perfect crystal}

The Hamiltonian Green's-function matrix $G(z)$ for the infinite crystal is most conveniently obtained from the LMTO-ASA equations

$$
\left(z 1-H^{\gamma}\right) G^{\gamma}(z)=1
$$

within the orthogonal representation. Here, $z$ is a complex energy and $H^{\gamma}$ the second-order LMTO Hamiltonian

$$
H^{\gamma}(\mathbf{k})=C+\sqrt{\Delta} S^{\gamma}(\mathbf{k}) \sqrt{\Delta}
$$

evaluated at a particular vector $\mathbf{k}$ in reciprocal space. The center $C$, bandwidth $\Delta$, and $\gamma$ potential parameters are taken from the self-consistent bulk calculation.

The structure-constant matrix $S^{\gamma}(\mathbf{k})$ that enters the LMTO Hamiltonian is obtained in a three-step process. First, we evaluate the tight-binding structure-constant matrix $S^{\beta}$ by means of the Dyson equation

$$
S_{R^{\prime} L^{\prime}, R L}^{\beta}=S_{R^{\prime} L^{\prime}, R L}^{0}+\sum_{R^{\prime \prime} L^{\prime \prime}} S_{R^{\prime} L^{\prime}, R^{\prime \prime} L^{\prime \prime}}^{0} \beta_{l^{\prime \prime}} S_{R^{\prime \prime} L^{\prime \prime}, R L}^{\beta}
$$

where $R$ indicate lattice sites and $L$ refers to the combined angular-momentum quantum numbers $(l, m)$ corresponding to real cubic harmonics. Explicit expressions for the conventional unscreened structure constants $S^{0}$ may be found in Refs. 9 and 15. The choice ${ }^{8}$

$$
\beta_{l}=\left\{\begin{array}{l}
0.3485, \quad l=s \\
0.05303, \quad l=p \\
0.0107, \quad l=d \\
0, \quad l>d
\end{array}\right.
$$

for the diagonal screening matrix $\beta$ leads to short-ranged structure constants, and hence the lattice summation in (3) extends only over a few shells of neighboring atoms. In the actual calculations we use the iterative procedure outlined in Sec. 4.10 of Andersen et al. ${ }^{9}$ Secondly, we perform the Bloch sum

$$
S_{R^{\prime} L^{\prime}, R L}^{\beta}(\mathbf{k})=\sum_{\mathbf{T}} e^{i \mathbf{k} \cdot \mathbf{T}} S_{R^{\prime} L^{\prime} ; R+T, L}^{\beta},
$$

where $R$ now runs only over the sites in the primitive cell and $\mathbf{T}$ are three-dimensional (3D) lattice translation vectors.

Finally, we transform to the orthogonal representation, obtaining the structure constants

$$
S^{\gamma}(\mathbf{k})=S^{\beta}(\mathbf{k})\left[1-(\gamma-\beta) S^{\beta}(\mathbf{k})\right]^{-1},
$$

which enter the eigenvalue equation

$$
\left[H^{\gamma}(\mathbf{k})-\epsilon \mathbf{1}\right] u^{\gamma}(\mathbf{k})=0
$$

with eigenvalues $\epsilon$ and eigenvectors $u^{\gamma}$. This procedure is efficient because the tight-binding structure constants $S^{\beta}$ can be evaluated once and for all for a given surface by short-ranged lattice summations in real space, because the Fourier transformation implied in the Bloch sum (5) makes the dimension of the matrix to be inverted in the transformation to the orthogonal representation (6) finite, and because (7) is a simple eigenvalue problem that can be solved by effective numerical methods.

The Green's-function matrix for the perfect crystal may now be obtained from

$$
G_{R^{\prime} L^{\prime}, R L}^{\gamma}(\mathbf{k}, z)=\sum_{j} \frac{u_{R^{\prime} L^{\prime}}^{\gamma j}(\mathbf{k})\left[u_{R L}^{\gamma j}(\mathbf{k})\right]^{*}}{z-\epsilon_{j}(\mathbf{k})}
$$


where $\epsilon_{j}(\mathbf{k})$ are the eigenvalues and $u_{R L}^{\gamma j}(\mathbf{k})$ the eigenvectors obtained by diagonalization of (7), i.e.,

$$
\left[u^{\gamma^{i}}(\mathbf{k})\right]^{\dagger}\left[C+\sqrt{\Delta} S^{\gamma}(\mathbf{k}) \sqrt{\Delta}\right] u^{\gamma j}(\mathbf{k})=\delta_{i j} \epsilon_{j}(\mathbf{k})
$$

$$
\left[u^{\gamma i}(\mathbf{k})\right]^{\dagger} u^{\gamma j}(\mathbf{k})=\delta_{i j}
$$

In a layer representation the Green's-function matrix becomes

$$
G_{R_{\perp}^{\prime} L^{\prime}, R_{\perp} L}^{\gamma}\left(\mathbf{k}_{\|}, z\right)=\frac{d_{\perp}}{2 \pi} e^{-i \mathbf{k}_{\|} \cdot\left(\mathbf{R}_{\|}-\mathbf{R}_{\|}^{\prime}\right)} \int_{0}^{2 \pi / d_{\perp}} d k_{\perp} e^{-i k_{\perp}\left(R_{\perp}-R_{\perp}^{\prime}\right)} G_{R^{\prime} L^{\prime}, R L}^{\gamma}(\mathbf{k}, z)
$$

where $d_{\perp}$ is a layer distance, $\mathbf{k}=\left(\mathbf{k}_{\|}, k_{\perp}\right)$, and $\mathbf{R}=$ $\left(\mathbf{R}_{\|}, R_{\perp}\right)$.

In the actual calculations the integral in (11) is evaluated by a procedure where the energy bands in the denominator and the phase factor times the eigenvector products in the nominator are linearized separately over suitably small $k_{\perp}$ intervals. The contributions from each interval are then obtained analytically by standard integrals of rational functions and summed numerically over the period $2 \pi / d_{\perp}$. This has proven to be a sufficiently accurate and efficient technique, especially because the Green's-function matrix need only be calculated once for a given surface. An even more efficient technique has recently been developed by Wenzien et $a l^{5}$

\section{KKR-ASA Green's function for the perfect crystal}

In the second step of our procedure, where we construct the Green's-function matrix for the semi-infinite crystal, it is important to work within a representation that has short range. Hence, it turns out to be most convenient to use the Green's-function matrix defined by the KKR-ASA equations in the tight-binding representation, i.e., by

$$
\left[P^{\alpha}(z)-S^{\alpha}(\mathbf{k})\right] g^{\alpha}(\mathbf{k}, z)=\mathbf{1}
$$

with $\alpha=\beta$. Here, the LMTO potential function $P^{\alpha}(z)$, which is essentially the cotangent to the phase shift, may be given by the parametrization

$$
P^{\alpha}(z)=\frac{\Gamma^{\alpha}}{V^{\alpha}-z}+\frac{1}{\gamma-\alpha}
$$

correct to second order in $\left(z-\epsilon_{\nu}\right)$, where $\epsilon_{\nu}$ is an arbitrary real energy, and the representation-dependent parameters are

$$
V^{\alpha}=C-\frac{\Delta}{\gamma-\alpha}, \quad \Gamma^{\alpha}=\frac{\Delta}{(\gamma-\alpha)^{2}}
$$

Within the orthogonal representation $(\alpha=\gamma)$ the potential function has a particularly simple parametrization, i.e.,

$$
P^{\gamma}(z)=\frac{z-C}{\Delta}
$$

and if this form is inserted into (12) and the result com- pared with the LMTO-ASA equations (1) we find the relation

$$
g^{\gamma}(z)=\sqrt{\Delta} G^{\gamma}(z) \sqrt{\Delta}
$$

between the Hamiltonian Green's-function matrix and the KKR-ASA Green's function matrix, valid to second order.

The transformation of the KKR-ASA Green's function between different representations has the form ${ }^{8,10}$

$$
g^{\beta}(z)=(\beta-\alpha) \frac{P^{\alpha}(z)}{P^{\beta}(z)}+\frac{P^{\alpha}(z)}{P^{\beta}(z)} g^{\alpha}(z) \frac{P^{\alpha}(z)}{P^{\beta}(z)},
$$

which only involves energy-dependent scalings. The Hamiltonian Green's-function matrix (11) for the perfect crystal may thus be transformed into the tight-binding KKR-ASA representation via

$$
g^{\beta}\left(\mathbf{k}_{\|}, z\right)=-\frac{z-V^{\beta}}{\Gamma^{\beta}}+\frac{z-V^{\beta}}{\sqrt{\Gamma^{\beta}}} G^{\gamma}\left(\mathbf{k}_{\|}, z\right) \frac{z-V^{\beta}}{\sqrt{\Gamma^{\beta}}},
$$

as obtained from (17) with $\alpha=\gamma,(15)$, and (13) with $\alpha=\beta$. This completes the construction of the Green'sfunction matrix for the perfect crystal, which forms the starting point for interface calculations.

\section{Perfect-vacuum Green's function}

In our implementation we have chosen to treat internal interfaces and surfaces by the same procedure. Hence, for a surface we "fill" the vacuum region with spheres which we take to be the same as those of the infinite crystal. The Green's-function matrix for the three-dimensionally infinite perfect vacuum may therefore in principle be obtained from free-electron potential parameters in exactly the same manner as desribed in the preceding section. This is, however, an inefficient procedure, and in practice we use an analytic expression, ${ }^{17}$ which we state below in a form valid for an energy $z$ in the complex plane cut along the positive real axis. This procedure allows one to form the $2 \mathrm{D}$ Bloch sum directly, thus avoiding the integration over $k_{\perp}$ otherwise needed to form the layerindexed Green's-function matrix.

The Green's function for the perfect vacuum in the conventional representation $(\alpha=0)$ is given in Ref. 17 as 


$$
g^{0}(z)=\frac{4}{s^{2}} W\{J(z), J(0)\}\left(S^{0}(z)-\frac{W\{K(z), J(0)\}}{W\{J(z), J(0)\}}\right) W\{J(z), J(0)\}
$$

where the Wronskians are defined by

$$
W\{f, g\}=s^{2}\left[f(s) g^{\prime}(s)-g(s) f^{\prime}(s)\right]
$$

and where the LMTO envelope functions are

$$
\begin{aligned}
& K_{l}(\kappa, r)=\left(\frac{i(\kappa w)^{l+1}}{(2 l-1) ! !}\right) h_{l}^{(1)}(\kappa r), \\
& J_{l}(\kappa, r)=\left(\frac{(2 l-1) ! !}{2(\kappa w)^{l}}\right) j_{l}(\kappa r) .
\end{aligned}
$$

In these expressions $\kappa=-\sqrt{z}$ where the principal root must be taken, $s$ is the radius of the atomic sphere, and $w$ is some dimension of the lattice, taken to be the average Wigner-Seitz radius. Finally, $h_{l}^{(1)}$ and $j_{l}$ are complex Hankel and Bessel functions, respectively.

The range of the irregular envelope function $K_{l}(\kappa, r)$ may be chosen to be exponentially short. Since

$$
h_{l}^{(1)}(\kappa r) \sim e^{i \kappa r}=e^{-i \sqrt{z} r}
$$

this requires that $\operatorname{Im} \kappa>0$, and hence that the $z$ plane is cut along the real axis. In the present application, where the Fermi energy is always below the vacuum level and the complex energy contour lies in the lower half-plane, both the imaginary and the real part of $z$ are negative, and the structure constants given in (23) below decay exponentially.

The energy-dependent conventional $(\alpha=0)$ structure constants máy be expressed in the two-center form ${ }^{18}$ with the $c$ axis chosen along the interatomic vector $R-R^{\prime}$ of length $d$, i.e.,

$$
S_{l^{\prime} l M}^{0}(\kappa d)=\sum_{l^{\prime \prime}}(i \kappa w)^{l+l^{\prime}-l^{\prime \prime}} g_{l^{\prime} l M}^{l^{\prime \prime}} K_{l^{\prime \prime}}(\kappa, d),
$$

where $g_{l_{l M}}^{l^{\prime \prime}}$ is essentially a spherical-harmonic Gaunt coefficient given by

$g_{l^{\prime} l M}^{l^{\prime \prime}}=(-1)^{l+1} \frac{2\left(2 l^{\prime \prime}-1\right) ! !}{(2 l-1) ! !\left(2 l^{\prime}-1\right) ! !} c^{l^{\prime \prime}}\left(l^{\prime} M, l M\right)$

$$
\begin{array}{r}
c^{l^{\prime \prime}}\left(l^{\prime} m^{\prime}, l m\right)=\left(\frac{4 \pi}{2 l^{\prime \prime}+1}\right)^{1 / 2} \int d \hat{r} Y_{L}(\hat{r}) Y_{L^{\prime}}^{*}(\hat{r}) Y_{L^{\prime \prime}}(\hat{r}), \\
l^{\prime \prime}=\left|l^{\prime}-l\right|,\left|l^{\prime}-l\right|+2, \ldots, l^{\prime}+l, \\
|M| \leq l^{\prime} \leq l, \quad m^{\prime}=m=M, \quad m^{\prime \prime}=m^{\prime}-m .
\end{array}
$$

For a general direction of the $c$ axis, e.g., the global $c$ axis of a crystal, we obtain the structure constant matrix from (23) and Table I in Ref. 18. Finally, we perform the 2D
Bloch sum, which converges rapidly, owing to the short range of the structure constants, and obtain the Green'sfunction matrix (19) directly in the $\mathbf{k}_{\|}$representation.

The tight-binding Green's-function matrix for a vacuum region is now simply given by

$$
g^{\beta}\left(\mathbf{k}_{\|}, z\right)=\beta \frac{P^{0}(z)}{P^{\beta}(z)}+\frac{P^{0}(z)}{P^{\beta}(z)} g^{0}\left(\mathbf{k}_{\|}, z\right) \frac{P^{0}(z)}{P^{\beta}(z)},
$$

where the scaling analogous to (18) is

$$
\frac{P^{0}}{P^{\beta}}=\left(\frac{\gamma}{\gamma-\beta}+\frac{\beta \Gamma^{\beta}}{V^{\beta}-z}\right)^{-1}
$$

and $V^{\beta}, \Gamma^{\beta}$, and $\gamma$ are free-electron potential parameters for the chosen $\epsilon_{\nu}(<0)$.

\section{Green's function for the semi-infinite crystal}

Equation (12) for the layer-indexed KKR-ASA Green's-function matrix (18) for a perfect crystal may be written in the block form

$$
\begin{array}{r}
\left(\begin{array}{cc}
P_{A}^{X}-S_{A A} & -S_{A B} \\
-S_{B A} & P_{B}^{X}-S_{B B}
\end{array}\right)\left(\begin{array}{ll}
g_{A A}^{X} & g_{A B}^{X} \\
g_{B A}^{X} & g_{B B}^{X}
\end{array}\right) \\
=\left(\begin{array}{ll}
1_{A A} & 0_{A B} \\
0_{B A} & 1_{B B}
\end{array}\right) .
\end{array}
$$

Here, the superscript $X$ refers to the atomic species of the perfect crystal for which the potential function is calculated, and the subscripts $A$ and $B$ refer to the layers on the left- and right-hand sides of the interface, respectively. The tight-binding superscript $\beta$ has been suppressed on the potential functions, the structure constants, and the Green's-function matrices.

Now, we cut the crystal in two by putting $S_{A B}=0$ and want to find the Green's-function matrix for the semiinfinite left-hand side, i.e., for the system with the KKR matrix $P_{A}^{X}-S_{A A}$. This is

$$
\tilde{g}_{A A}^{X}=\left(P_{A}^{X}-S_{A A}\right)^{-1},
$$

which we shall presently express in terms of matrix elements of the perfect-crystal Green's function (18). To perform the inversion (28) we make use of the fact that the structure constants have a limited range, so that

$$
S_{A B}=0 \text { for }\{A B\} \neq\{a b\}
$$

where $\{a b\}$ is a small subset of layers close to the interface. By inserting (28) into (27) and writing out the first row we find

$$
\begin{aligned}
& \left(\tilde{g}_{A A}^{X}\right)^{-1} g_{A A}^{X}-S_{A b} g_{b A}^{X}=1_{A A}, \\
& \left(\tilde{g}_{A A}^{X}\right)^{-1} g_{A b}^{X}-S_{A b} g_{b b}^{X}=0_{A b},
\end{aligned}
$$

which upon elimination of $S_{A b}$ now leads to the desired expression, 


$$
\tilde{g}_{A A}^{X}=g_{A A}^{X}-g_{A b}^{X}\left(g_{b b}^{X}\right)^{-1} g_{b A}^{X},
$$

for the Green's-function matrix for the left-hand side of the semi-infinite crystal. Similarly, one finds for the righthand side with atomic species $Y$,

$$
\tilde{g}_{B B}^{Y}=g_{B B}^{Y}-g_{B a}^{Y}\left(g_{a a}^{Y}\right)^{-1} g_{a B}^{Y}
$$

It follows that any matrix element of the Green's function for the idealized semi-infinite crystal may be found by inversion of a single finite matrix, the dimensions of which are given by the "hopping" range of the tight-binding structure constants.

The above derivation of the expression for the semiinfinite Green's-function matrix is a particular application of Löwdin ${ }^{19}$ downfolding, which in the present context was termed the "ideal construction" by Williams et al. ${ }^{20}$ Similar procedures were derived by Lannoo ${ }^{21}$ for point defects and by Velickỳ and Kurdnovskỳ ${ }^{22}$ for surfaces.

\section{Interface LMTO Green's function}

At this stage, from the band calculations and the subsequent transformations detailed in the previous sections we know the Green's-function matrix defined by

$$
\begin{gathered}
\left(\begin{array}{cc}
P_{A}^{X}-S_{A A} & 0_{A B} \\
0_{B A} & P_{B}^{Y}-S_{B B}
\end{array}\right)\left(\begin{array}{cc}
\tilde{g}_{A A}^{X} & 0_{A B} \\
0_{B A} & \tilde{g}_{B B}^{Y}
\end{array}\right) \\
=\left(\begin{array}{ll}
1_{A A} & 0_{A B} \\
0_{B A} & 1_{B B}
\end{array}\right)
\end{gathered}
$$

which is simply the Löwdin-downfolded Green's-function matrices [(32) and (33)] for the two noninteracting semiinfinite crystals taken together.

The desired Green's-function matrix for the combined system may be written

$$
\begin{array}{r}
\left(\begin{array}{cc}
P_{A}^{X}-S_{A A} & -S_{a b} \\
-S_{b a} & P_{B}^{Y}-S_{B B}
\end{array}\right)\left(\begin{array}{ll}
g_{A A} & g_{A B} \\
g_{B A} & g_{B B}
\end{array}\right) \\
=\left(\begin{array}{ll}
1_{A A} & 0_{A B} \\
0_{B A} & 1_{B B}
\end{array}\right)
\end{array}
$$

where we have reintroduced the tight-binding structure constants that couple the two sides of the interface. A comparison between the last two equations shows that (35) may be obtained from the Dyson equation

$$
g=\tilde{g}+\tilde{g}\left(\begin{array}{cc}
0_{a a} & S_{a b} \\
S_{b a} & 0_{b b}
\end{array}\right) g
$$

which within the double layer $\{a, b\}$ becomes a finite matrix equation. If this is written out block for block we find

$$
\begin{aligned}
& g_{a a}=\tilde{g}_{a a}+\tilde{g}_{a a} S_{a b} g_{b a}, \\
& g_{b a}=\tilde{g}_{b b} S_{b a} g_{a a}
\end{aligned}
$$

and substitution of $g_{b a}$ into (37) leads to

$$
g_{a a}=\left(1_{a a}-\tilde{g}_{a a} S_{a b} \tilde{g}_{b b} S_{b a}\right)^{-1} \tilde{g}_{a a} .
$$

Similarly, we find for the other sub-blocks

$$
\begin{aligned}
g_{a b} & =\tilde{g}_{a a} S_{a b} g_{b b}, \\
g_{b b} & =\left(1_{b b}-\tilde{g}_{b b} S_{b a} \tilde{g}_{a a} S_{a b}\right)^{-1} \tilde{g}_{b b},
\end{aligned}
$$

and hence we have the complete Green's-function matrix inside the interface region defined by the "hopping" range of the tight-binding structure constants.

The interface Green's function derived above is given in terms of quantities obtained from band calculations on perfect crystals of the atomic species $X$ and $Y$. This means, in particular, that the potential functions retain their bulk values right up to the interface. For a real system the relaxation $\Delta P^{i}=P^{i}-P$ that occurs in the potential functions close to the interface may be taken into account by means of the finite Dyson equation

$$
g^{i}=g+g \Delta P^{i} g^{i}
$$

giving the interface Green's-function matrix in terms of the unrelaxed $g$. In the actual calculations it is (42) which, together with the Poisson equation, is solved repeatedly until self-consistency is achieved in potentials and charge densities.

\section{B. The charge density}

At each iteration one needs to evaluate the charge density on the basis of the one-electron spectrum. In the present technique we obtain the valence charge density from the one-center expression

$$
n_{R}^{v}\left(\mathbf{r}_{R}\right)=\sum_{L^{\prime} L^{\prime \prime}} Y_{L^{\prime}}\left(\hat{\mathbf{r}}_{R}\right)^{*}\left(\int^{E_{F}} d \epsilon \phi_{R l^{\prime}}^{\gamma}\left(\epsilon, r_{R}\right) \mathcal{D}_{R L^{\prime}, R L^{\prime \prime}}(\epsilon) \phi_{R l^{\prime \prime}}^{\gamma}\left(\epsilon, r_{R}\right)\right) Y_{L^{\prime \prime}}\left(\hat{\mathbf{r}}_{R}\right)
$$


where $\mathbf{r}_{R}=\mathbf{r}-\mathbf{R}$ is inside the atomic sphere at $R, Y_{L}(\hat{\mathbf{r}})$ is a cubic harmonic, $\mathcal{D}_{R L^{\prime}, R L^{\prime \prime}}(\epsilon)$ is the partial-wave decomposed state density as derived from the Green's function [see (48) below], and where the partial wave itself $\phi_{R l}^{\gamma}(r)$ is normalized in the atomic sphere of radius $S_{R}$, i.e.,

$$
\int_{0}^{S_{R}} \phi_{R l}^{\gamma}(\epsilon, r)^{2} r^{2} d r=1
$$

If the first three terms of the Taylor expansion

$$
\begin{aligned}
\phi_{R l}^{\gamma}(\epsilon, r)= & \phi_{\nu R l}^{\gamma}(r)+\left(\epsilon-\epsilon_{\nu}\right) \dot{\phi}_{\nu R l}^{\gamma}(r) \\
& +\frac{1}{2}\left(\epsilon-\epsilon_{\nu}\right)^{2} \ddot{\phi}_{\nu R l}^{\gamma}(r)+\cdots
\end{aligned}
$$

around $\epsilon_{\nu}$ are inserted into (43) and a core charge density is added, the total charge density inside the sphere at $R$ may be found from the cubic-harmonic expansion

$$
\begin{aligned}
& n_{R}\left(\mathbf{r}_{R}\right)=\sum_{L} n_{R L}^{v}\left(r_{R}\right) Y_{L}\left(\hat{\mathbf{r}}_{R}\right)+n_{R}^{c}\left(r_{R}\right) Y_{s}\left(\hat{\mathbf{r}}_{R}\right) \\
& n_{R L}^{v}\left(r_{R}\right)=\sum_{L^{\prime} L^{\prime \prime}} C_{L L^{\prime} L^{\prime \prime}} f_{R L^{\prime} L^{\prime \prime}}\left(r_{R}\right) \\
& C_{L L^{\prime} L^{\prime \prime}}=\int d \hat{r} Y_{L}^{*}(\hat{r}) Y_{L^{\prime}}^{*}(\hat{r}) Y_{L^{\prime \prime}}(\hat{r})
\end{aligned}
$$

where to second order in $\epsilon-\epsilon_{\nu}$ and neglecting the index $\nu R$ on the radial functions

$$
\begin{aligned}
f_{R L^{\prime} L^{\prime \prime}}\left(r_{R}\right)= & \phi_{l^{\prime}}^{\gamma}\left(r_{R}\right) \phi_{l^{\prime \prime}}^{\gamma}\left(r_{R}\right) m_{R L^{\prime} L^{\prime \prime}}^{00}+\dot{\phi}_{l^{\prime}}^{\gamma}\left(r_{R}\right) \dot{\phi}_{l^{\prime \prime}}^{\gamma}\left(r_{R}\right) m_{R L^{\prime} L^{\prime \prime}}^{11}+\phi_{l^{\prime}}^{\gamma}\left(r_{R}\right) \dot{\phi}_{l^{\prime \prime}}^{\gamma}\left(r_{R}\right) m_{R L^{\prime} L^{\prime \prime}}^{01}+\dot{\phi}_{l^{\prime}}^{\gamma}\left(r_{R}\right) \phi_{l^{\prime \prime}}^{\gamma}\left(r_{R}\right) m_{R L^{\prime} L^{\prime \prime}}^{10} \\
& +\frac{1}{2} \phi_{l^{\prime}}^{\gamma}\left(r_{R}\right) \ddot{\phi}_{l^{\prime \prime}}^{\gamma}\left(r_{R}\right) m_{R L^{\prime} L^{\prime \prime}}^{02}+\frac{1}{2} \ddot{\phi}_{l^{\prime}}^{\gamma}\left(r_{R}\right) \phi_{l^{\prime \prime}}^{\gamma}\left(r_{R}\right) m_{R L^{\prime} L^{\prime \prime}}^{20}
\end{aligned}
$$

and the moments of the state density are given by

$$
\begin{aligned}
m_{R L^{\prime} L^{\prime \prime}}^{q^{\prime} q^{\prime \prime}} & =\int^{E_{F}} d \epsilon\left(\epsilon-\epsilon_{\nu R l^{\prime}}\right)^{q^{\prime}} \mathcal{D}_{R L^{\prime}, R L^{\prime \prime}}(\epsilon)\left(\epsilon-\epsilon_{\nu R l}\right)^{q^{\prime \prime}} \\
& =\frac{1}{2 \pi i} \oint d z\left(z-\epsilon_{\nu R l^{\prime}}\right)^{q^{\prime}} G_{R L^{\prime}, R L^{\prime \prime}}^{\gamma}(z)\left(z-\epsilon_{\nu R l}\right)^{q^{\prime \prime}}
\end{aligned}
$$

In the last equation we have used the residue theorem in conjunction with the fact that the eigenstates of the interface are the poles of the Hamiltonian Green's function, and hence, the contour should enclose all the occupied states and cut the real axis at the Fermi level. However, since the Green's-function matrices obey the relation

$$
G_{R^{\prime} L^{\prime}, R L}^{\gamma}(z)^{\dagger}=G_{R^{\prime} L^{\prime}, R L}^{\gamma}\left(z^{*}\right)
$$

as one may deduce from the spectral representation (8), the contour may be reduced to a semicircle in the lower half-plane and, e.g.,

$$
\begin{aligned}
\oint d z G_{R^{\prime} L^{\prime}, R L}^{\gamma}(z) & \\
& =\int_{U} d z G_{R^{\prime} L^{\prime}, R L}^{\gamma}(z)-\left(\int_{U} d z G_{R^{\prime} L^{\prime}, R L}^{\gamma}(z)\right)^{\dagger} .
\end{aligned}
$$

The Green's-function matrices which enter the contour integral are found by the transformation

$$
G^{\gamma}\left(\mathbf{k}_{\|}, z\right)=\frac{1}{z-V^{\beta}}+\frac{\sqrt{\Gamma^{\beta}}}{z-V^{\beta}} g^{\beta}\left(\mathbf{k}_{\|}, z\right) \frac{\sqrt{\Gamma^{\beta}}}{z-V^{\beta}}
$$

inverse to (18). In general, the extra poles introduced at $V^{\beta}$ might fall within the contour, and hence one might worry that the evaluation of the moments could be invalidated. However, close to $V^{\beta}$ the tight-binding Green'sfunction matrix behaves as

$$
g^{\beta} \approx-\frac{z-V^{\beta}}{\Gamma^{\beta}}
$$

whereby the effect of the poles cancel and the contour integral is left unchanged.

In order to solve Poisson's equation for the interface, and also to calculate the electrostatic contributions to the total energy, one needs the multipole moments of the charge density. These are calculated from

$$
\begin{aligned}
Q_{R}^{L} & \equiv \frac{\sqrt{4 \pi}}{2 l+1} \int_{R} d \mathbf{r} Y_{L}(\hat{\mathbf{r}})^{*}\left(\frac{r}{S_{R}}\right)^{l} n_{R}(\mathbf{r})-Z_{R} \delta_{l, s} \\
& =\frac{\sqrt{4 \pi}}{2 l+1} \sum_{L^{\prime} L^{\prime \prime}} C_{L L^{\prime} L^{\prime \prime}} \int^{S_{R}} d r\left(\frac{r}{S_{R}}\right)^{l} r^{2} f_{R L^{\prime} L^{\prime \prime}}(r)-z_{R} \delta_{l, s}
\end{aligned}
$$


where $Z_{R}$ is the atomic number, $z_{R}$ the valence, and $C_{L L^{\prime} L^{\prime \prime}}$ are cubic-harmonic Gaunt coefficients.

\section{The one-electron potential}

Once the charge density is obtained, the electrostatic part of the one-electron potential in the spheres may be found as a solution to Poisson's equation. This solution can be written in the one-center form

$$
V_{C}(\mathbf{r})=\sum_{R L} V_{C ; R L}\left(r_{R}\right) Y_{L}\left(\hat{\mathbf{r}}_{R}\right)
$$

i.e., as an atom-centered cubic-harmonic expansion. In the ASA we use only the spherically symmetric, i.e., $L=$ $s$ part of the potential, and hence the potential in the atomic sphere at $R$ in the two-dimensional unit cell is approximated by

$$
V_{C ; R}\left(r_{R}\right)=V_{C ; R s}\left(r_{R}\right) Y_{s}+\frac{1}{S} \sum_{R^{\prime} L^{\prime}} M_{R R^{\prime}}^{s L^{\prime}} Q_{R^{\prime}}^{L^{\prime}}
$$

where the intrasphere contribution is obtained by numerical integration of the radial Poisson equation

$$
\left(\frac{d^{2}}{d r^{2}}+\frac{2}{r} \frac{d}{d r}\right) V_{C ; R s}\left(r_{R}\right)=-8 \pi n_{R s}\left(r_{R}\right),
$$

with the boundary condition

$$
V_{C ; R s}\left(S_{R}\right) Y_{s}=\frac{2 Q_{R}^{s}}{S_{R}}
$$

and where the intersphere contribution is given by a multipole expansion, the coefficients of which are given in the following section.

It is the monopole, dipole, and quadrupole terms which govern the long-range behavior of the electrostatic potential, and for many systems, e.g., impurities in metals, it is sufficient to include only the monopole contribution, i.e., the Madelung potential, to the last term in (55). However, in two-dimensional systems, where the charge density may be rather nonspherical, it is important to include also the dipole, i.e., $L^{\prime}=p_{z}$, contribution to the multipole potential in order to ensure correct behavior at infinity. Thus, in the present implementation the dipole potential barrier has two contributions, one from the monopoles, i.e., the net charges in the spheres, and one from the dipole charges in the spheres. These contributions are of the same order of magnitude but have opposite signs, and it is only when they are combined in the self-consistency procedure that one obtains an accurate estimate of the dipole barrier.

With these assumptions the one-electron potential in the sphere at $R$ is

$$
V_{R}\left(r_{R}\right)=-\frac{2 Z_{R}}{r_{R}}+V_{C ; R}\left(r_{R}\right)+\mu_{\mathrm{xc}}\left[n_{R s}\left(r_{R}\right) Y_{s}\right]
$$

where the first term is the potential from the nuclei and the last term the exchange-correlation potential in the local-density approximation. ${ }^{23}$

\section{Multipole potential}

The evaluation of the multipole matrices $M_{R R^{\prime}}^{s L^{\prime}}$ that enter (55) requires a $2 \mathrm{D}$ Ewald technique in analogy with the usual $3 \mathrm{D}$ case. We have developed such a technique based on the complete solution to the 3D Poisson equation, assuming spherically symmetric Gaussian charge densities distributed on planes parallel to the interface. For the two most important matrices we find

$$
\begin{aligned}
M_{R R^{\prime}}^{s s}= & \frac{4 \pi S}{A}\left(\left(R_{\perp}^{\prime}-R_{\perp}\right) \operatorname{erfc}\left\{\lambda\left(R_{\perp}^{\prime}-R_{\perp}\right)\right\}-\frac{1}{\lambda \sqrt{\pi}} e^{-\lambda^{2}\left(R_{\perp}^{\prime}-R_{\perp}\right)^{2}}\right) \\
& +2 S\left(\frac{\pi}{A} \sum_{\mathbf{k}_{\|} \neq \mathbf{0}} \frac{1}{k_{\|}} V_{R_{\perp} R_{\perp}^{\prime}}\left(k_{\|}\right) e^{i \mathbf{k}_{\|} \cdot\left(\mathbf{R}_{\|}-\mathbf{R}_{\|}^{\prime}\right)}+\frac{1}{\sqrt{\pi}} \sum_{\mathbf{t}_{\|}} \frac{\Gamma\left(\frac{1}{2}, \lambda^{2} T^{2}\right)}{T}-\frac{2 \lambda}{\sqrt{\pi}} \delta_{R_{\perp}, R_{\perp}^{\prime}}\right) \\
M_{R R^{\prime}}^{s p_{z}}= & 4 \sqrt{3} S^{2}\left(-\frac{\pi}{2 A} \sum_{\mathbf{k}_{\|}} V_{R_{\perp} R_{\perp}^{\prime}}^{-}\left(k_{\|}\right) e^{i \mathbf{k}_{\|} \cdot\left(\mathbf{R}_{\|}-\mathbf{R}_{\|}^{\prime}\right)}+\frac{1}{\sqrt{\pi}} \sum_{\mathbf{t}_{\|}} \frac{\Gamma\left(\frac{3}{2}, \lambda^{2} T^{2}\right)}{T^{2}} \frac{R_{\perp}-R_{\perp}^{\prime}}{T}\right)
\end{aligned}
$$

where $S$ is the average Wigner-Seitz radius in all space, $A$ the surface area of the 2D unit cell, $\lambda$ an Ewald convergence parameter, and where the prime on the summation sign indicates that $T=0$ should be excluded from the sum. In addition, we have defined

$$
\begin{aligned}
V_{R_{\perp} R_{\perp}^{\prime}}^{ \pm}\left(k_{\|}\right)= & e^{k_{\|}\left(R_{\perp}-R_{\perp}^{\prime}\right)} \operatorname{erfc}\left(\frac{k_{\|}}{2 \lambda}+\lambda\left(R_{\perp}-R_{\perp}^{\prime}\right)\right) \\
& \pm e^{-k_{\|}\left(R_{\perp}-R_{\perp}^{\prime}\right)} \operatorname{erfc}\left(\frac{k_{\|}}{2 \lambda}-\lambda\left(R_{\perp}-R_{\perp}^{\prime}\right)\right)
\end{aligned}
$$

and

$$
\mathbf{T}=\mathbf{t}_{\|}+\mathbf{R}^{\prime}-\mathbf{R}
$$

where $\mathbf{t}_{\|}$is a translation vector of the $2 \mathrm{D}$ lattice.

Parry $^{24}$ has obtained an expression for the $M^{s s}$ Madelung matrix similar to (59) by integrating the usual Ewald expression over the third dimension. However, his result is only valid for systems where each individual layer is neutral, and does not include electrostatic dipole-barrier terms. In contrast, the present result only 
assumes charge neutrality in the entire two-dimensional unit cell, and hence gives the correct description of the interface dipole barrier.

\section{Charge neutrality}

At each iteration the charge density in the $2 \mathrm{D}$ cell is estimated from the Green's function and the partial waves. Since the total charge derived in this manner may in general not be neutral, charge neutrality must be imposed at each iteration. In a conventional self-consistent energy-band calculation, charge neutrality is established by shifting the Fermi level until a known state density accommodates the appropriate number of electrons. This procedure cannot be used in an interface calculation because the Fermi level is determined by the perfect crystal(s), and because the number of electrons is obtained from a contour integral, not from a state density. Instead, we apply a site-independent shift to the potentials in the interface region, and hence to $\Delta P$ in the Dyson equation (42). The shift converges rapidly and is found to be very small.

\section{The dipole barrier and the work function}

In the solution to Poisson's equation for a semi-infinite metal occupying $-\infty<r_{\perp} \leq 0$ one may choose the envelope function of the electrostatic potential, as given by the Madelung term in (55), to be zero deep inside the metal, i.e., $\bar{V}_{C}\left(r_{\perp} \rightarrow-\infty\right)=0$. In that case, the electrostatic dipole barrier across the surface may be obtained from (55), (59), and (60), and we find

$$
\begin{aligned}
\Delta \phi & =\bar{V}_{C}\left(r_{\perp} \rightarrow \infty\right)-\bar{V}_{C}\left(r_{\perp} \rightarrow-\infty\right) \\
& =\frac{1}{S}\left(\frac{8 \pi S^{2}}{A}\right) \sum_{R}\left(Q_{R}^{s} \frac{R_{\perp}}{S}+\sqrt{3} Q_{R}^{p_{z}}\right) .
\end{aligned}
$$

In the ASA, the envelope function of the electrostatic potential is the potential from the monopoles and dipoles at the sphere centers. For a 3D crystal this envelope potential is chosen to be periodic and of zero average, i.e., $\bar{V}_{C}(\mathbf{r})=0$, which is then taken as the zero of energy

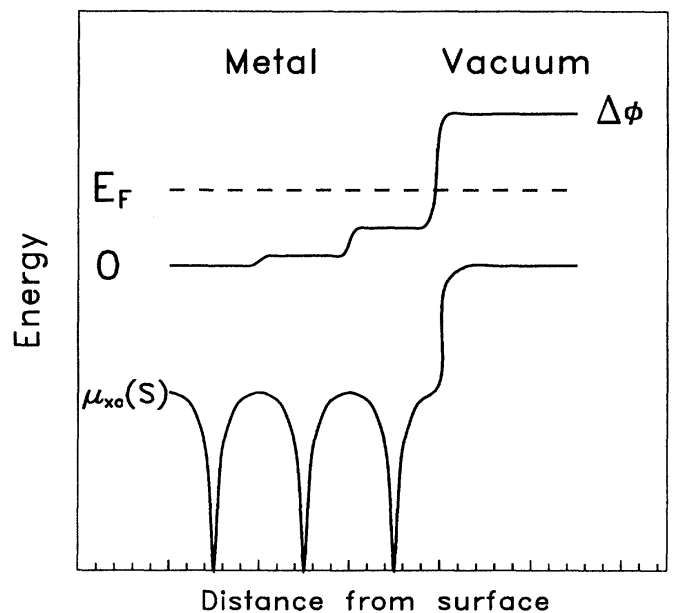

FIG. 1. Schematic representation of the electrostatic dipole potential near a metal surface (top), and the effective one-electron potential where the dipole contribution has been subtracted (bottom). The full one-electron potential is the sum of the two potentials shown.

in an ASA band-structure calculation. For a 3D crystal with one sphere per cell this energy zero coincides with the electrostatic potential at the surface of the atomic sphere, and the work function of the corresponding semiinfinite metal may be estimated from

$$
W=\Delta \phi-E_{F}
$$

where $E_{F}$ is the Fermi level for the $3 \mathrm{D}$ crystal with the ASA energy zero. In Fig. 1 we have sketched the potential near the surface of a metal and indicated the relevant energies.

\section{E. The total energy}

In the Born-Oppenheimer, local-density, atomicsphere, and frozen-core approximations, the total energy of the interface may be expressed in terms of the quantities already defined as $^{25}$

$$
\begin{aligned}
E= & \sum_{R} T_{R}^{v}+\frac{1}{2 S} \sum_{R L, R^{\prime} L^{\prime}} Q_{R}^{L} M_{R R^{\prime}}^{L L^{\prime}} Q_{R^{\prime}}^{L^{\prime}}-\sum_{R} \frac{1}{\sqrt{4 \pi}} \int_{R} d^{3} r n_{R s}^{v}(r) \frac{2 Z_{R}}{r}+\sum_{R} \frac{1}{4 \pi} \int_{R} d^{3} r n_{R s}^{v}(r)\left[\frac{1}{2} V_{C ; R s}^{v}(r)+V_{C ; R s}^{c}(r)\right] \\
& +\sum_{R} \frac{1}{\sqrt{4 \pi}} \int_{R} d^{3} r n_{R s}(r) \epsilon_{x c}\left[n_{R s}(r) Y_{s}\right]-\sum_{R} \frac{1}{\sqrt{4 \pi}} \int_{R} d^{3} r n_{R s}^{c}(r) \epsilon_{x c}\left[n_{R s}^{c}(r) Y_{s}\right] .
\end{aligned}
$$

Here, the $R$ summation runs over those atoms inside the 2D unit cell that are included in Dyson's equation (42), and the atomic-sphere projected kinetic energy may be obtained from

$$
\begin{aligned}
T_{R}^{v}= & \sum_{L}\left(\epsilon_{\nu R l} m_{R L L}^{00}+m_{R L L}^{10}\right) \\
& -\frac{1}{\sqrt{4 \pi}} \int_{R} d^{3} r n_{R s}^{v}(r) V_{R}(r) .
\end{aligned}
$$


In (66) the charges and the electrostatic potentials have been separated into valence and core contributions, indicated by the superscripts $v$ and $c$, respectively. In addition, we have neglected terms that only pertain to the (frozen) core. Hence, when we calculate surface energies
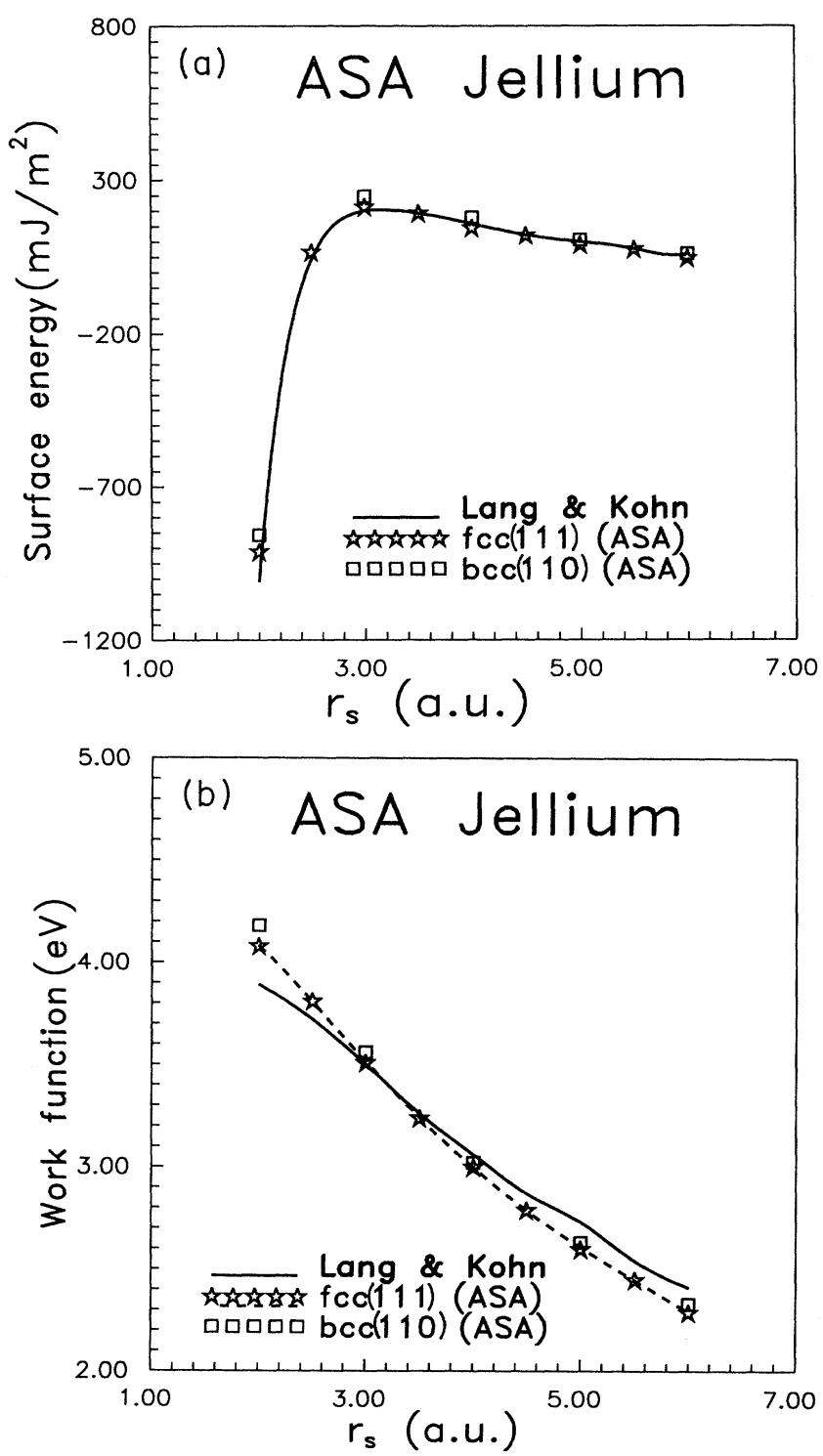

FIG. 2. Comparison of values of the surface energy (a) and the work function (b) calculated within the uniform positive background (jellium) model. The solid line gives the results obtained by Lang and Kohn (Refs. 26 and 27). Asterisks and squares give the results of the LMTO-ASA Green's-function technique for the two close-packed surfaces fcc (111) and bcc (110), respectively. In the LMTO-ASA calculations the charge perturbation was confined to four layers of spheres on the metal side and two layers of spheres on the vacuum side. The sphere radii were chosen equal to $r_{s}$ such that each sphere within the bulk contained one electron. All calculations, including those of Lang and Kohn, were performed within the exchange-correlation approximation of Wigner (Ref. 28). as the difference in total energy of bulk and surface, we shall assume that the same terms have been neglected in the calculations for the perfect crystal.

\section{ASA JELLIUM, A TEST CASE}

The surface of a simple metal may be approximated by a model in which the conduction electrons move in a uniform neutralizing semi-infinite charge density. This so-called jellium model was first solved self-consistently within local-density theory by Lang and Kohn, ${ }^{26} 27$ and their calculated work functions and surface energies, reproduced in Fig. 2, provide a test of the accuracy of our Green's-function method.

In the ASA, we approximate a jellium surface by layers of overlapping atomic spheres, each charged with a uniform neutralizing background density to simulate the metal side of the surface, and layers of atomic spheres with no background charge to simulate vacuum. This approximation models a warped surface, and we would expect the calculated work functions and surface energies to be close to the conventional jellium results only for close-packed surfaces such as fcc (111) and bcc (110).

In Fig. 2 we present surface energies and work functions for two close-packed jellium surfaces calculated over a wide range of densities by means of the LMTO-ASA Green's-function technique. In these calculations, the sphere size was such that the $3 \mathrm{D}$ jellium contained one electron per sphere, i.e., $S=r_{s}$, and to be consistent with earlier calculations we used the exchange-correlation approximation due to Wigner. ${ }^{28}$ The close agreement between the results of these calculations and those of the conventional jellium model shows that the present LMTO-ASA calculations are well converged, that the LMTO-ASA method is accurate, and that the ASA jellium is a good appoximation to the exact planar jellium model.

This agreement with Lang and Kohn's jellium results is extremely satisfying because we would expect a jellium surface to be the strongest possible test of the LMTOASA method and because we have implemented and applied the method in a particularly simple form. That is, potentials and charge densities are assumed to be spherically symmetric except for the dipole contribution $M_{R R^{\prime}}^{s p_{z}}$ included in the potential (55) and total energy (66). Hence, as far as surface energies and work functions are concerned there seems to be no need to go beyond the atomic-sphere approximation.

\section{THE ALKALI METALS}

For many years the alkali metals have served as a testing ground for surface calculations and exchangecorrelation approximations starting with Lang and Kohn's jellium calculations ${ }^{26,27}$ and presently culminating in the recent work by Zhang et al. ${ }^{29}$ who cite most of the previous results. The basis of most of these calculations has been the jellium model, to which was added the 
effect of the ion lattice treated either within first-order perturbation theory or by a variational approach. ${ }^{30}$

The outcome of the jellium-type calculations for real alkali metals may be judged from Fig. 3, where we present surface energies and work functions plotted as functions
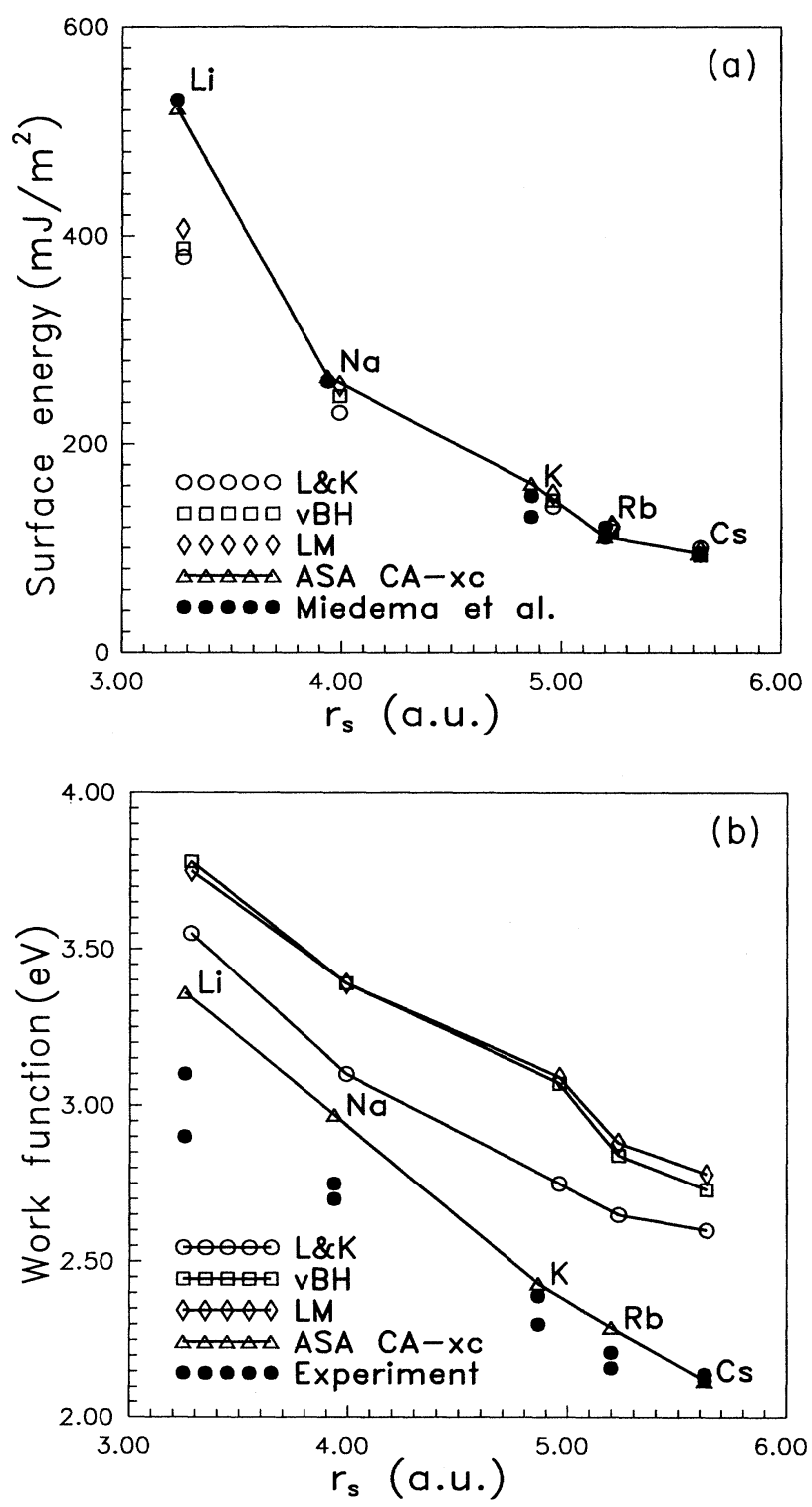

FIG. 3. Calculated surface energies (a) and work functions (b) compared with experimental values. Circles give the original Lang and Kohn results (Refs. 26 and 27) including an ion-lattice correction; triangles give LMTO-ASA results with the Ceperley-Alder (Refs. 31 and 32) exchangecorrelation approximation. Squares and diamonds give results from Zhang et al. (Ref. 29) with von Barth-Hedin (Ref. 33) and Langreth-Mehl (Ref. 34) exchange-correlation functionals, respectively. Solid circles give (a) surface energies derived from surface tensions (Refs. 35 and 36) and (b) experimental work functions (Refs. 37 and 38 ). of the density variable $r_{s}$. It is seen that the jellium-based models give surface energies that are in good agreement with values derived from the measured surface tensions of the liquid metals, except for $\mathrm{Li}$, where the surface energy is underestimated by $25 \%$. Similarly, they lead to
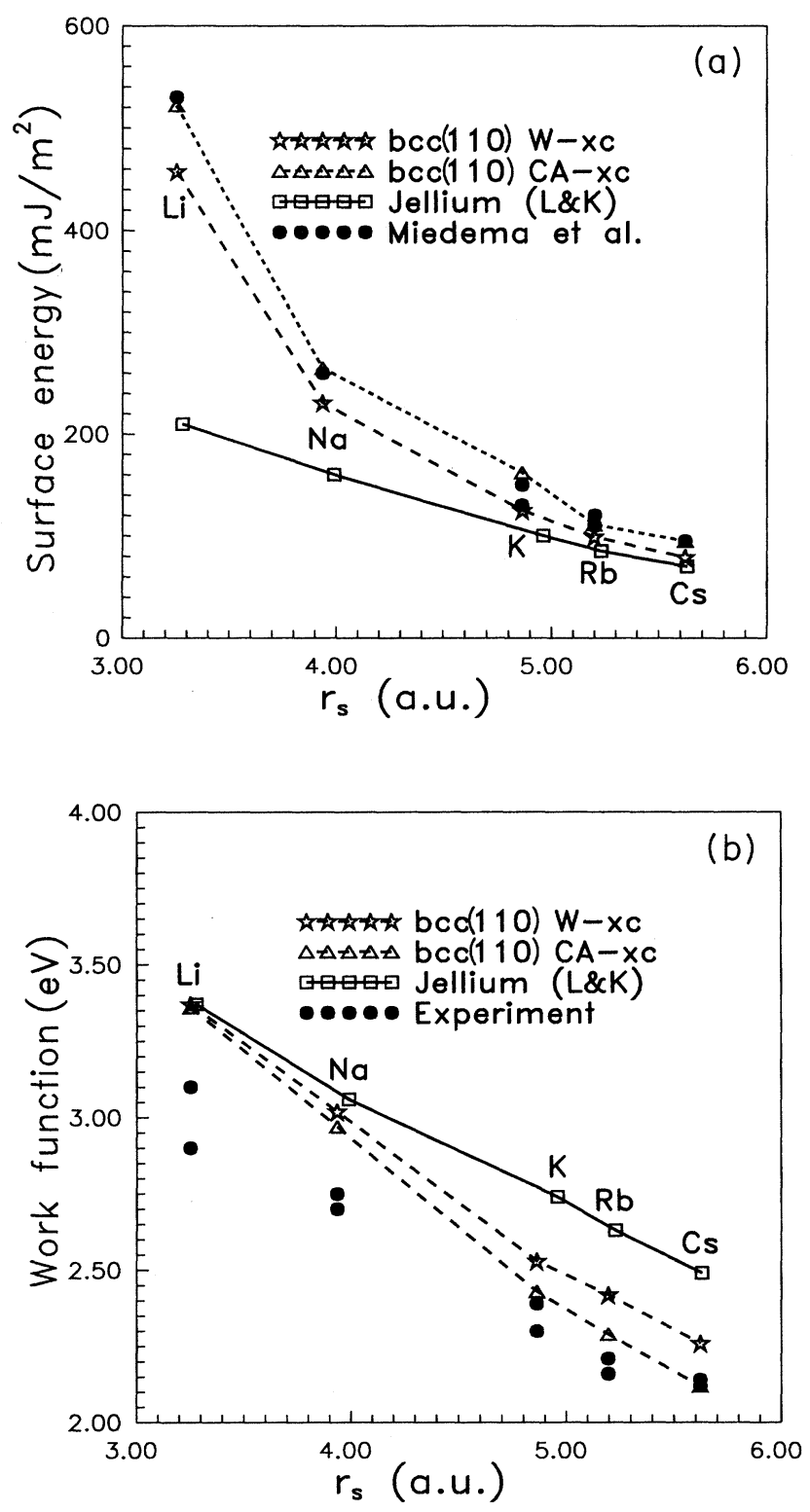

FIG. 4. Calculated surface energies (a) and work functions (b) compared with experimantal values. Squares give the Lang and Kohn jellium results (Refs. 26 and 27) without the ion-lattice corrections, asterisks and triangles give LMTO-ASA results with Wigner (Ref. 28) and CeperleyAlder (Refs. 31 and 32) exchange-correlation functionals, respectively. Solid circles give (a) surface energies derived from surface tensions (Refs. 35 and 36 ) and (b) experimental work functions (Refs. 37 and 38). 
work functions, which, over the whole density range, are typically $30 \%$ larger than the experimental values.

We have used the LMTO-ASA Green's-function technique in conjunction with various exchange-correlation functionals to calculate surface energies and work functions for the alkali metals. In Fig. 3 we show the results obtained with the exchange-correlation functional of Ceperley and Alder, ${ }^{31}$ as parametrized by Perdew and Zunger ${ }^{32}$ which we have found to give the best overall agreement with experiment. It is seen that the combined effect of an accurate treatment of the ion lattice and the choice of exchange-correlation functional leads to close agreement with the experimentally derived surface energies, as well as the experimental work functions for all alkali-metals, the main deviation being an overestimate of the work functions for $\mathrm{Li}$ and $\mathrm{Na}$ by approximately $10 \%$.

If one compares the Lang and Kohn jellium calculations without the ion-lattice correction reproduced in Fig. 4 to LMTO-ASA results obtained with Wigner and with Ceperley-Alder functionals one may assess the effect of the ion lattice and the choice of exchange-correlation approximation separately. It is seen that the lattice contribution to the surface energy, Fig. 4(a), is considerable at high densities (low $r_{s}$ ) and insignificant at low densities. In contrast to this, the difference between the Wigner and the Ceperley-Alder surface energies is essentially constant through the alkali-metal series. For the work functions, Fig. 4(b), it is seen that both the lattice contribution and the effect of the choice of exchangecorrelation approximation are large at small densities and insignificant at high densities.

A comparison between Figs. 3(a) and 4(a) shows that perturbation theory as well as the single-parameter variational approach ${ }^{30}$ used by Zhang et al. ${ }^{29}$ provide good approximations for the lattice contributions to the surface energy for the less dense metals $\mathrm{Na}, \mathrm{K}, \mathrm{Rb}$, and $\mathrm{Cs}$ but underestimate the contribution in $\mathrm{Li}$ by $30 \%$. For the work functions, Figs. 3(b) and 4(b), one finds that both perturbation theory and the variational approach, in contrast to the LMTO-ASA, lead to positive lattice contributions, and hence to significantly overestimated values.

At densities corresponding to $\mathrm{Li}$ and $\mathrm{Na}$ our LMTO-ASA calculations with Ceperley-Alder exchangecorrelation overestimate the work functions by $10 \%$, see Fig. 4(b). A comparison with Fig. 2(b) indicates that the LMTO-ASA gives accurate jellium work functions in this density range, and for that reason the overestimate might be attributed to a failure of the Ceperley-Alder exchangecorrelation approximation at high densities. On the other hand, the LMTO-ASA overestimates the jellium work function at high densities, and since the density in real $\mathrm{Li}$ and $\mathrm{Na}$ is not uniform, and in certain parts of space will exceed $r_{s}$ values of 3 , the failure might be attributed to the ASA.

\section{SUMMARY}

This paper reports the implementation of a selfconsistent Green's-function technique for calculating ground-state properties of large classes of interfaces. The ground state is found within the local-density approximation, and the Green's-function technique is based on the linear-muffin-tin-orbitals method in the tight-binding representation. The atomic-sphere approximation is used for the potential, but for the charge density a dipole contribution is included.

The jellium model is used as a test of the accuracy of the method, and we find that the LMTO-ASA technique yields surface energies and work functions, which over a large density range are in extremely good agreement with earlier jellium calculations.

In a series of calculations for the bcc (110) surface of the alkali metals we find surface energies that are in very good agreement with zero-temperature values derived from surface tensions of the liquid metals. In addition, we find that for $\mathrm{K}, \mathrm{Rb}$, and Cs (low densities) the calculated work functions are in close agreement with the experimental values. For $\mathrm{Li}$ and $\mathrm{Na}$ (higher densities) the work functions are overestimated by $10 \%$. This may be attributed to a failure in either the local-density approximation or the atomic-sphere approximation.

\section{ACKNOWLEDGMENTS}

This work was begun while one of us (H.L.S.) was at the Max-Planck-Institute in Stuttgart, and it is a great pleasure to thank O. K. Andersen, W. Lambrecht, and O. Jepsen for scientific support during the development of the Green's-function technique. H.L.S. also benefitted greatly from discussion with $M$. Springborg while at NORDITA. Finally, we wish to thank J. Nørskov and $K$. Jacobsen for sharing their insights in surface physics with us, and O. H. Nielsen for maintaining an efficient and user-friendly computational environment. This work was supported by a grant from the Novo Foundation.
${ }^{1}$ D. Kalkstein and P. Soven, Surf. Sci. 26, 85 (1971).

${ }^{2}$ J. E. Inglesfield, J. Phys. C 4, L14 (1971).

${ }^{3}$ J. Pollmann and S. T. Pantelides, Phys. Rev. B 18, 5524 (1978).

${ }^{4}$ G. A. Benesh and J. E. Inglesfield, J. Phys. C 17, 1595 (1984).
${ }^{5}$ B. Wenzien, J. Kudrnovskỳ, V. Drchal, and M. Šob, J. Phys. Condensed Matter 1, 9893 (1989).

${ }^{6} \mathrm{~J}$. E. Inglesfield and G. A. Benesh, Phys. Rev. B 37, 6682 (1988).

7 J. M. MacLaren, S. Crampin, D. D. Vvedensky, and J. Pendry, Phys. Rev. B 40, 12164 (1989). 
${ }^{8}$ O. K. Andersen and O. Jepsen, Phys. Rev. Lett. 53, 2571 (1984).

${ }^{9}$ O. K. Andersen, O. Jepsen, and D. Glötzel, in Highlights of Condensed-Matter Theory, edited by F. Bassani, F. Fumi, and M. P. Tosi (North-Holland, New York, 1985).

${ }^{10}$ O. K. Andersen, Z. Pawlowska, and O. Jepsen, Phys. Rev. B 34, 5253 (1986).

${ }^{11} \mathrm{O}$ Gunnarsson, O. Jepsen, and O. K. Andersen, Phys. Rev. B 27, 7144 (1983).

${ }^{12}$ F. Beeler, M. Scheffler, O. Jepsen, and O. Gunnarsson, Phys. Rev. Lett. 54, 2525 (1985).

${ }^{13}$ F. Beeler, O. K. Andersen, and M. Scheffler, Phys. Rev. Lett. 55, 1498 (1985).

${ }^{14}$ W. Lambrecht and O. K. Andersen, Surf. Sci. 178, 256 (1986); private communication.

${ }^{15}$ O. K. Andersen, Phys. Rev. B 12, 3060 (1975).

${ }^{16}$ H. L. Skriver, The LMTO Method (Springer, Berlin, 1984).

${ }^{17}$ See Appendix B of Ref. 11.

${ }^{18}$ J. C. Slater and G. F. Koster, Phys. Rev. 94, 1498 (1954).

${ }^{19}$ P. O. Löwdin, J. Chem. Phys. 19, 1396 (1951).

${ }^{20}$ A. R. Williams, P. J. Feibelman, and N. D. Lang, Phys. Rev. B 26, 5433 (1982).

${ }^{21}$ M. Lannoo, Ann. Phys. (Paris) 3, 391 (1968).

${ }^{22}$ B. Velickỳ and J Kurdnovskỳ, Surf. Sci. 64, 411 (1977).

${ }^{23}$ See, e.g., O. Gunnarsson and B. I. Lundqvist, Phys. Rev. B 13, 4274 (1976).
${ }^{24}$ D. E. Parry, Surf. Sci. 49, 433 (1975).

${ }^{25}$ See, e.g., Ref. 11, Sec. II D.

${ }^{26}$ N. D. Lang and W. Kohn, Phys. Rev. B 1, 4555 (1970).

${ }^{27}$ N. D. Lang and W. Kohn, Phys. Rev. B 3, 1215 (1971).

${ }^{28}$ E. P. Wigner, Phys. Rev. 46, 1002 (1934).

${ }^{29}$ Z. Y. Zhang, D. C. Langreth, and J. P. Perdew, Phys. Rev. B 41, 5674 (1990).

${ }^{30}$ R. Monnier and J. P. Perdew, Phys. Rev. B 17, 2595 (1978); 22, 1124(E) (1980).

${ }^{31}$ D. M. Ceperley and B. J. Alder, Phys. Rev. Lett. 45, 566 (1980).

${ }^{32}$ J. Perdew and A. Zunger, Phys. Rev. B 23, 5048 (1981).

${ }^{33}$ U. von Barth and L. Hedin, J. Phys. C 5, 1629 (1972).

${ }^{34}$ D. C. Langreth and M. J. Mehl, Phys. Rev. B 28, 1809 (1983); 29, 2310(E) (1983).

${ }^{35}$ A. R. Miedema and B. E. Nieuwenhuys, Surf. Sci. 104, 491 (1981).

${ }^{36}$ F. R. de Boer, R. Boom, W. C. M. Mattens, A. R. Miedema, and A. K. Niessen, Cohesion in Metals (NorthHolland, New York, 1988), Table IV-2.

${ }^{37}$ D. Germer and H. Mayer, Z. Phys. 210, 391 (1968).

${ }^{38} \mathrm{H}$. B. Michaelson, in CRC Handbook of Chemistry and Physics, edited by R. C. Weast, M. J. Astle, and W. H. Beyer, 64th ed. (Chemical Rubber, Cleveland, 1983), p. E-76. 